Revue d'histoire de l'Amérique française

DEVVUE D.HISTOIRE DE L'AMÉRIQUE FRANÇAISE

\title{
Aspects de la psychologie du groupe de pression ultramontain canadien-français (1870-1890)
}

\section{Louis Chevrette}

Volume 25, numéro 2, septembre 1971

URI : https://id.erudit.org/iderudit/303063ar

DOI : https://doi.org/10.7202/303063ar

Aller au sommaire du numéro

Éditeur(s)

Institut d'histoire de l'Amérique française

ISSN

0035-2357 (imprimé)

1492-1383 (numérique)

Découvrir la revue

Citer cet article

Chevrette, L. (1971). Aspects de la psychologie du groupe de pression ultramontain canadien-français (1870-1890). Revue d'histoire de l'Amérique française, 25(2), 155-189. https://doi.org/10.7202/303063ar d'utilisation que vous pouvez consulter en ligne. 


\title{
ASPECTS DE LA PSYCHOLOGIE DU GROUPE DE PRESSION ULTRAMONTAIN CANADIEN-FRANÇAIS (1870-1890)*
}

\author{
Louis Chevrette \\ Université Laval \\ Québec
}

\section{A. Introduction}

Cet article veut informer des résultats d'une recherche empirique opérée par un essai d'analyse thématique de 60 messages écrits. A titre de préliminaires et afin qu'on évalue mieux la validité scientifique des résultats, nous fournirons quelques indications historico-génétiques, descriptives et critiques sur la source imprimée où ont été puisés les 60 messages observés. L'analyse thématique que nous en avons faite a voulu répondre à deux objectifs de recherche qui seront secondement précisés. Puis nous définirons notre concept d'idéologie et sa définition opératoire mis en cause dans la quête du premier de ces objectifs. Nous légitimerons ensuite notre choix de 60 messages fait dans les Voix canadiennes pour satisfaire à nos deux objectifs de recherche. L'analyse thématique est une technique d'observation systématique des contenus de communications verbales. Des chercheurs américains l'ont d'abord conçue et développée. Nous décrirons enfin cette technique avant de livrer les résultats de son application.

\section{B. Préliminaires}

1. La source exploitée: notice historico-génétique, descriptive et critique

* Le présent communiqué est tiré de notre thèse de maîtrise ès arts (histoire) : Idéologie, traits culturels, plan de réaction, perception et motivation du groupe de pression ultramontain canadien-français (18701890) (Université Laval, 1971, lxvii-215 p.). Il présente les résultats d'un essai d'application d'un cadre conceptuel et théorique à une analyse historique. L'appareil conceptuel est celui de l'école parsonienne américaine. Particulièrement opératoire pour la recherche historique, à notre sens, il fut à l'origine exprimé dans une terminologie anglaise plutôt hermétique. Comme des sociologues francophones l'ont déjà fait, nous avons dû la traduire et à cette fin former quelques néologismes d'un goût discutable. Le lecteur nous en excusera.

RHAF, vol. 25, no 2 (septembre 1971) 
De 1908 à 1922, l'homme de lettres et libraire-éditeur ${ }^{1}$ français Arthur Savaète publia à Paris un ouvrage de 12 tomes intitulé Voix canadiennes, Vers l'abîme. Savaète fut aussi l'auteur de cette série qui totalise quelque 6,066 pages.

C'est après la mort de son "ami intime", Mgr Justin Fèvre, survenue le 30 août $1907^{2}$, que Savaète décida d'écrire ses Voix canadiennes ${ }^{3}$. Ce prélat historien français ${ }^{4}$ avait de son vivant "mis sur le chantier" deux ouvrages: une "Histoire du Canada" et une "Vie de Mgr Laflèche, Evêque des Trois-Rivières". Mais "contretemps irréparable, (...) il laissait en mourant l'un et l'autre inachevés" 5. En publiant ses Voix canadiennes, Savaète voulait donner suite, d'une certaine façon, à ces œuvres ébauchées. $^{6}$

Pour ce faire, il disait posséder des "copies" des "importants documents inédits, même secrets" que le défunt prélat avait accumulés "sur les affaires du Canada"7 depuis $1888 .{ }^{8} \mathrm{Il}$ se vantait aussi d'avoir pu finalement repérer, après la mort de Mgr Fèvre, les "collaborateurs" canadiens "informés et sagaces" qui l'avaient ainsi "secrètement" documenté. ${ }^{9}$ Par leur entremise, il avait pu se procurer "d'autres (documents) qui ne le cèdent en rien pour l'intérêt qu'ils méritent". 10

1 Ainsi est définie la fonction-statut d'Arthur Savaète, dans D. Jordell, rédacteur, Catalogue général de la librairie française. Continuation de l'ouvrage d'Otto Lorentz (Paris, 1911), t. 22, I: 459. Là-même on apprend que Savaète est né dans le département du Nord (France) en 1858.

2 Pierre Savard, Jules-Paul Tardivel, la France et les Etats-Unis, 1851-1905 (Québec, Presses de l'Université Laval, 1967), 451, note 257. (A l'avenir: Tardivel). Voix).

3 Arthur Savaète, Voix canadiennes, Vers l'abîme, I: 1-7. (A l'avenir:

4 Dans Pierre Savard, Tardivel, $143-165$ et $450-457$, on trouve une description de certains traits de la vie et de l'œuvre de Mgr Justin Fèvre, ultramontain polémiste et historien fort prolixe qui fut curé de Louze en France de 1854 à 1907. M. Savard nous apprend en outre les relations qu'entretint le prélat avec Jules-Paul Tardivel et d'autres ultramontains canadiens-français. Mgr Fèvre exerça aussi une action ultramontaine au Canada français par ses articles parus dans la Vérité (Québec) et l'Etendard (Trois-Rivières) de 1888 à 1889 et par sa publication d'une biographie controversée de Jules-Paul Tardivel en 1906.

5 Arthur Savaète, Voix, I: 5 .

6 Ibid., 5-6.

7 Ibid., II : 12.

8 Pierre Savard, Tardivel, 450.

9 Arthur Savaète, Voix, II : 13-14.

10 Ibid., I: 4. 
L'édition d'une telle documentation rassemblée par l'auteurlibraire-éditeur constitue essentiellement le contenu des Voix canadiennes. Tous les textes qu'on y trouve ont été rédigés dans le feu de cette série d'interactions ${ }^{11}$ antagoniques survenues dans la seconde moitié du $\mathrm{XIX}^{\mathbf{e}}$ siècle entre des membres laïcs et clercs des groupements "ultramontain" et "libéral" canadienfrançais. ${ }^{12}$ Les acteurs ultramontains de ces conflits ont signé à peu près tous les écrits, pour la plupart des lettres ou mémoires privés restés jusque-là inédits et inconnus du public canadienfrançais.

Savaète les publiait “à ses risques et périls" surtout pour réhabiliter dans la culture canadienne-française la mémoire des évêques Bourget et Laflèche, respectivement de Montréal et des Trois-Rivières ${ }^{13}$. Selon lui, l'historiographie des Canadiens français asservie aux intérêts des "libéraux" depuis leur prise des pouvoirs politiques québécois et canadien, n'avait pas encore rendu justice à ces prélats, grands leaders du mouvement ultramontain canadien-français, des années 1840 à $1890{ }^{14}$. "Depuis bientôt dix lustres années", cette historiographie "libérale" avait, "dans un intérêt inavouable, dissimulé, soigneusement écarté, joyeusement entouré de mystère et de silence tant de documents

11 Nous n'attachons pas au terme "interaction" un concept identique à celui qu'a défini Robert Mandrou dans La France aux XVIIe et XVIIIe siècles (Paris, Presses Universitaires de France, 1967), 62, 303, 304, 305 et 308. Si nous comprenons M. Mandrou, il y entendit par "interaction": "jeux d'influences réciproques", "rapports dialectiques", "mouvements aller et retour" entre cette "double réalité", vaguement conceptualisée et classifiée, qu'il dénomma tantôt "l'Ancien Régime socio-économique et l'Ancien Régime socio-culturel", tantôt "cultures et rapports sociaux et économiques", tantôt "l'économique et le mental", tantôt "la base économique sur laquelle se fonde la représentation sociale" ou, autrement dit, "les conditionnements socio-économiques et les positions idéologiques, les horizons intellectuels et affectifs". M. Mandrou a noté que son concept d" "interaction" sera difficilement opératoire. Pour nous, "interaction" a le sens que lui ont donné Talcott Parsons et al., dans le cadre conceptuel de leur théorie générale de l'action, cadre et théorie qu'ils ont communiqués sans circonlocutions dans Toward a General Theory of Action (Cambridge, Harvard University Press, 1951), 31. Une "interaction" y est conceptualisée comme une activité humaine corporelle ou psychique qui est reliée d'une façon ou d'une autre par des principes de relations (l'objet des sciences sociales selon les auteurs) à des objets extérieurs à l'organisme qui produit cette activité, que ces objets soient sociaux ou non.

12 Robert Rumilly donne des récits détaillés de ces conflits çà et là dans son Histoire de la Province de Québec (Montréal, Editions Bernard Valiquette, 1940), I: 1-6.

13 Arthur Savaète, Voix, IV : 5.

14 Ibid., IV : 2-3. 
historiques". Elle avait "massacré à la bonne franquette, en dehors des règles de l'art, (...) Mgr Ignace Bourget, de respectable mémoire, (...) Mgr Laflèche, l'abbé Pelletier et tant d'autres". ${ }^{15}$ Elle avait surfait "l'irrésistible cardinal Taschereau et ses satellites de l'Université Laval ameutés contre la justice, le bon droit et les saines doctrines romaines". ${ }^{16}$

Mais l' "historien impartial et serviteur intègre et désintéressé de la vérité" que Savaète disait être, entrecoupait la publication de ses documents de celle de ses propres commentaires. Dans un langage fort expressif, il dépeignait personnes, groupes et interactions canadiens du passé et du présent et les évaluait dépendamment des modèles et croyances ultramontains dont l'institutionnalisation, de son aveu, le motivait farouchement.

Savaète s'est gardé de dévoiler la provenance canadienne exacte de ce qu'il affirmait être d' "irréfutables documents historiques". Nous avons néanmoins retracé les originaux d'une dizaine d'entre eux dans des dépôts d'archives canadiens ${ }^{17}$. Nous avons vérifié que l'édition de Savaète était conforme aux originaux. On ne saurait douter qu'il en fut ainsi de l'édition de tous les textes des Voix canadiennes. ${ }^{18}$

Nous avons eu en mains les cinq premiers tomes de la série. Une première lecture rapide nous a amené à utiliser une partie des pièces qui y sont publiées pour une recherche à deux objectifs connexes.

\section{Objectifs de notre recherche}

Le premier objectif fut a), de préciser le contenu de deux types d'assemblage d'idées qui, très vraisemblablement, furent institutionnalisés chez à peu près tous les Canadiens français ${ }^{19}$ des années 1870 et 1880 et b), de vérifier l'existence chez eux

15 Ibid., III : 1.

16 Ibid., III : 1.

17 Soit aux Archives du Séminaire des Trois-Rivières et aux Archives de l'Archevêché de Québec.

18 On trouvera, dans notre thèse, les détails de la preuve d'authenticité établie pour lever ce doute.

19 On a présentement une connaissance historique assez imprécise du nombre variable et des noms des personnes qui ont composé ce groupement durant son existence. L'histoire de sa formation et de sa disparition, de la structuration des rôles et statuts de ses membres et de leurs interactions ne nous est connue que dans ses grandes lignes. La thèse inédite d'H. L. Robertson, The Ultramontane Group in French Canada, 1867-1886 (maîtrise ès arts, Université Queen's (Ontario), 1952) est, à notre connaissance, la seule étude qui traite spécifiquement de ces aspects du groupement dans l'une de ses parties. 
d'un troisième type d'assemblage d'idées et d'en préciser éventuellement le contenu. Ces trois catégories d'ensemble de propositions recherchées furent: 1) l'idéologie du groupement ultramontain; 2) les éléments de sa culture (modèles normatifs et croyances légitimatives) qui ont orienté la partie évaluative de l'idéologie ; 3) un plan d'une proréaction évaluative lié à celle-ci. Les propositions idéologiques, culturelles et stratégiques communiquées dans les écrits d'ultramontains qui eurent un statut élevé dans leur groupe nous sont mal connues ${ }^{20}$. Elles n'en ont pas moins, sans nul doute, motivé et orienté les interactions évaluatives morales ${ }^{21}$ accomplies par les membres du groupement

Cette partie est très largement inspirée de l'Histoire de la Province de Québec de Robert Rumilly, I-VI, et de son Mgr Laflèche et son temps (Montréal, Editions du Zodiaque, 1938). Robertson n'a donc pratiquement rien ajouté de neuf, si ce n'est quelques assertions hypothétiques, à l'apport de Rumilly qu'il faut souvent croire sur parole. Dans Tardivel, Pierre Savard a ajouté, entre autres, quelques éléments nouveaux à notre connaissance de ces aspects du groupement, notamment à celle des interactions de ses membres et de leurs relations "internationales".

${ }^{20}$ L'institutionnalisation d'une idéologie, telle qu'on la définira infra, dans les personnalités du noyau du groupement ultramontain des années 1870 et 1880 ne nous faisait pas difficulté à l'origine de notre recherche. H. L. Robertson l'avait établie dans The Ultramontane Group in French Canada, 1867-1886. La thèse inédite de Robertson est d'ailleurs, que nous sachions, la seule étude qui ait présenté une esquisse de ce que fut le contenu des éléments culturels - les croyances métaphysiques surtout - qui ont orienté la partie évaluative de l'idéologie. Robertson n'a cependant pas étudié l'ordination de celle-ci. De plus, il n'a analysé, pour la percevoir, que les journaux du groupement ultramontain.

Il y avait possibilité, avons-nous cru en concevant le premier objectif de notre analyse, que le type de documents étudiés par Robertson ait présenté au lecteur des croyances idéologiques ou culturelles perçues fausses par les ultramontains et qui aient été antinomiques à des croyances perçues vraies. Les articles des journaux ultramontains, on le sait, n'étaient pas destinés qu'à des lecteurs ultramontains. Au contraire, ils visaient presque tous à persuader des journalistes éditorialistes opposés aux idées ultramontaines.

Pour découvrir les croyances du groupement ultramontain, nous avons observé des documents autres que ses journaux. Différents de ceux-ci dans leur contenu et destinés, pour une partie d'entre eux, à d'autres ultramontains, ces documents nous feraient peut-être connaître, avons-nous pensé, l'existence de croyances ultramontaines perçues vraies, s'ils nous montraient des antinomies apparemment volontaires entre des croyances que nous trouverions et certaines de celles trouvées par Robertson. De ce point de vue surtout, comme de celui de pouvoir ajouter d'autres croyances pas nécessairement contradictoires à celles trouvées par Robertson et de connaître des perceptions de personnes et une stratégie des ultramontains, notre recherche a voulu être complémentaire de la sienne.

21 Pour une définition du concept de ce type d'action dite évaluative morale, voir Talcott Parsons, The Social System (Glencoe, Ill., The Free Press, 1951), 45-58. 
ultramontain canadien-français dans les années 1870 et 1880 . Ces interactions, des historiens se sont plu à les narrer, après les avoir reconnues et parfois réexpliquées avec des concepts et modèles plus ou moins folkloriques formés au siècle dernier ou antérieurement, quand ce n'est parfois même avant la fondation de la Sorbonne, s'il faut en croire le dictionnaire Robert ${ }^{22}$.

Le second objectif de notre recherche a été de prendre un aperçu sommaire des perceptions plus ou moins communes qu'a eues de lui-même et d'autrui ${ }^{23}$ le groupe de pression ultramontain durant la période 1870-1890. La connaissance des résultats de recherches empiriques en psychologie sociale nous avait appris que la sélectivité et la distortion des perceptions de personnes faites par un individu ou une collectivité sont généralement fortement corréliées à ses modèles culturels normatifs et/ou motivations ${ }^{24}$. Afin surtout de vérifier l'existence d'une corrélation entre d'une part la motivation évaluative morale commune qui solidarisait les ultramontains québécois de

${ }^{22} \mathrm{~A}$ l'aide de concepts et modèles plus ou moins populaires formés au siècle dernier ou antérieurement, à l'aide des postulats indémontrables des métaphysiques contemporaines et de leurs taxonomies tripartites stratifiées, inopératoires, mal intégrées et réifiées (v.g., l" "économique", le "social" et le "mental"), reconceptualiser et réexpliquer une réalité qui n'est plus par le biais de lectures distraites de communications aléatoirement choisies, tel serait-il le processus cognitif dit "de gros bon sens" ou "strictement empirique" de certains historiens et sociologues contemporains ? Ce processus re-cognitif, François Simiand crut l'avoir observé en 1903, comme l'atteste l'article qu'il publia alors dans la Revue de Synthèse historique, t. 6, 16 (1903) : 13-19. L'article s'intitulait: "Méthode historique et science sociale, Etude critique d'après les ouvrages récents de M. Lacombe et de M. Seignobos".

23 Perception de personne est ici entendu au sens large que lui donnent généralement les psychologues sociaux et signifie aperception ou connaissance résultant du processus de la perception proprement dit - processus qui peut lui-même, comme on l'a expérimentalement démontré, être constitué d'activités psychiques telles que jugements, reconnaissances, inférences, classifications - Prise dans ce sens large, une perception n'implique pas nécessairement, de la part du sujet apercevant, une expérience directe de la personne perçue. Les évaluations des traits perçus ne sont généralement pas incluses dans cette catégorie "perception": elles définissent des "attitudes" subséquentes aux "perceptions". Une perception de personne ou groupe ne peut être verbalement exprimée, bien entendu, que par des jugements de réalité ou encore des catégories lexicales.

24 Notons, parmi bien d'autres publications qui ont rendu compte de ces recherches, celles de J. S. Bruner et C. C. Goodman, "Value and Need as Organizing Factors in Perception", Journal of Abnormal Social Psychology, 42 (1947): 33-34; A. Pepitone, "Motivational Effects in Social Perceptions", Human Relations, 3 (1944) : 57-76; H. Fensterheim et M. E. Tresselt, "The Influence of Value Systems on the Perception of People", Journal of Abnormal Social Psychology, 5 (1955) : 629-636. 
1870-1890 et d'autre part 1) les caractères d'une sélection historique et 2 ) peut-être aussi d'une distorsion historique vérifiable de quelques-unes de leurs aperceptions, il nous a paru intéressant de faire une grossière analyse d'un échantillon de celles-ci.

\section{Notre concept d'idéologie et sa définition opératoire}

\section{a) Le concept}

Nous avons formé de ce que nous appelons idéologie un concept que nous définissons comme suit 25 : une idéologie est une évaluation ${ }^{26}$ faite, en un temps donné, par une société, sous-collectivité ou personne, d'un élément normatif plus ou moins spécifique d'un système culturo-social perçu actualisé, institutionnalisé ou en voie de l'être. Celui ou ceux qui évaluent un tel système social peuvent l'actualiser, l'institutionnaliser, le promouvoir ou ne pas le faire. Il ou ils peuvent faire partie ou non de la société où ils perçoivent le système évalué. Celui-ci peut être un système social dominant, déviant ou variant, l'ensemble de ces systèmes sociaux, un ou des sous-systèmes constitutifs de l'un ou l'autre d'entre eux. De plus l'évaluation du système, croyons-nous, est nécessairement basée sur sa prise en conscience ou connaissance directe ou indirecte préalable, laquelle conscience est plus ou moins sélective, distordue et théorique. Une cognition-évaluation de système social ou idéologie est verbalement communiquée, privément ou publiquement. Sa partie évaluative est en outre orientée par un ou des modèles culturels normatifs plus ou moins spécifiques et la ou les croyances évaluatives qui les rationalisent. Ce ou ces modèles et croyances culturels motivent positivement celui ou ceux qui produisent l'idéologie. Mais nous ne les incluons pas dans notre concept afin de ne pas mêler inextricablement idéologies et

25 Pour une discussion des principaux concepts dont procède celui qui vient d'être défini, voir "Un modèle psychologique du processus du changement social et un concept d'idéologie y intégré", article paru dans Culture (mars 1971) : 405-414.

26 Une "évaluation" est ici conceptualisée différemment d'un "sentiment": un sentiment serait un phénomène cathectique verbalement traduit par une proposition du type "j'apprécie plus ou moins tel objet"; une évaluation serait un phénomène cognitif dépendant d'un phénomène évaluatif (formation de standards). Elle serait verbalement communiquée par une proposition du type "telle action est plus ou moins bonne ou mauvaise", "tel objet est plus ou moins esthétique ou laid", "telle croyance est plus ou moins vraie ou fausse".

La cause d'une idéologie ainsi conçue ne saurait toujours être un "stress", une idéologie pouvait être une évaluation positive aussi bien que négative. Bien qu'il conceptualise une idéologie comme une évaluation positive ou négative, Parsons conçoit qu'elle est toujours causée par des tensions ou "strains". Son hypothèse paraît plutôt invraisemblable. 
cultures; celles-là portent toujours, du reste, surr un ou des éléments normatifs de celles-ci ${ }^{27}$.

b) La définition opératoire

Une idéologie ainsi conceptualisée serait verbalement traduite par un assemblage plus ou moins complexe: 1) de propositions évaluatives dont le ou les référents seraient un ou des systèmes culturo-sociaux perçus institutionnalisés ou en voie de l'être; et 2) de propositions existentielles aux mêmes référents. Identifier des idéologies empiriques ou des sections d'idéologies ne ferait donc pas difficulté. Il suffirait d'observer ces deux types de propositions dites ou écrites, explicitement ou implicitement en un temps donné, par un ou des membres d'une société ou de les leur faire dire ou écrire par des questionnaires ou entrevues.

La définition qu'on vient de prendre du concept 1) d'idéologie et celle que nous utilisons des concepts 2) d'éléments culturels (modèles normatifs ${ }^{28}$ et croyances évaluatives ${ }^{29}$ ) orienteurs d'é-

27 Ainsi conçues, les idéologies ne sauraient donc être qu'un reflet (il faudrait définir ce terme de façon opératoire) d'une structure sociale ou d'une "infrastructure économique". Elles ne sauraient non plus "combler les indéterminations de ces structures, leur donner cohérence ou fixer des objectifs d'actions", ni être "partie prenante aux mécanismes sociaux" comme on les conçoit dans Recherches sociographiques, X, 2-3 (mai-décembre 1969) : 145. Dans le cadre conceptuel parsonien, c'est la culture qui est le modèle ou le reflet et la rationalisation d'un système (ou structure) social actualisé ou institutionnalisé. L'idéologie est la prise de conscience d'un ou plusieurs modèles culturels ou de ses rationalisations et leur évaluation. Un modèle culturel peut être, selon son degré de généralité, une valeur, une norme, une collectivité, un rôle.

28 Pour des définitions précises et opératoires des types de modèles normatifs (valeur, norme, collectivité, rôle), voir Talcott Parsons, "An Outline of the Social System", Theories of Society, Foundations of Modern Sociological Theory (New York, Free Press of Glencoe, 1961), 42-44. C'est sans réticence que nous avons employé le mot valeur et le concept qu'il recouvre, contrairement à ce que dit faire Fernand Dumont dans Le lieu de l'homme, La culture comme distance et mémoire (Montréal, Editions HMH, 1968), 10. Ce concept de valeur, Clyde Kluckhohn et al. l'avaient longuement décrit avec définition opératoire en 1951 dans "Values and Value-Orientations in The Theory of Action: An Exploration in Definition and Classification", in Toward a General Theory of Action, 388-433. Talcott Parsons a spécifié le concept et en a restreint l'étendue en 1961: du concept de Kluckhohn et al., il a formé quatre classes qu'il a appelées valeur, norme, collectivité, rôle.

Le concept de Kluckhohn nous paraît opératoire. Que le terme valeur “appartienne à un vocabulaire qui n'est sans doute pas sans relation avec l'érosion des cultures concrètes", que "la transcendance hâtive qu'il semble suggérer rejoigne les illusions du spiritualisme de naguère" est peut-être possible, mais reste à démontrer.

29 Nous donnons à ce que nous dénommons "croyances évaluatives" le 
valuations idéologiques et 3) de plan de proréaction ${ }^{30}$ nous ont amené à délimiter comme suit le premier objectif de notre recherche: il consisterait à essayer de préciser 1) le contenu spécifique fondamental de ces trois types d'assemblage d'idées très probablement institutionnalisées dans les personnalités des membres du groupement ultramontain québécois des années $1870-1890 ; 2$ ) les changements qu'aurait pu subir pareil contenu durant la même durée; 3) l'ordination (classements et mises en relation) faite par les ultramontains des éléments constitutifs du contenu.

\section{Justification du choix de 60 documents fait dans les Voix canadiennes}

Nous avons sélectionné, dans les Voix canadiennes, une soixantaine de documents qui, avons-nous cru, constituaient un ensemble valable pour répondre à nos deux objectifs de recherche $:^{31}$ les dates de la rédaction de tels documents s'étalent à peu près également des années 1870 à 1890 ; leurs auteurs repré-

sens que donnait Parsons en 1951, dans The Social System, à ce qu'il appelait alors idéologie. Le concept serait opératoire pour l'observation des contenus de communications verbales. Pour y reconnaître une proposition existentielle qui fut ou est légitimative pour son auteur, il faudrait, croyons-nous, y constater également: 1) l'existence d'au moins une proposition spécifiant une action faite ou à faire par son auteur ou d'autres (du type "j'ai fait ceci ou cela" ou "il (me ou nous) faut faire ceci ou cela") et : 2) la mise en relation de cette proposition par un lien causal explicite ou implicite ("parce que") avec la proposition existentielle qui de ce fait sera dite légitimative.

Cette double constatation est d'ailleurs, selon nous, la seule façon de discerner sûrement dans les paroles d'un individu ou d'une collectivité l'attitude premièrement évaluative qu'il peut ou a pu avoir à l'endroit d'une croyance qu'il communique.

30 Dans "Toward a Classification of Interactions", Toward a General Theory of Action, 439-440, Henry A. Murray définit une "proaction" et une "proréaction" comme une série intégrée d'actions humaines dirigées vers un but éloigné dans le temps et dénomme "stratégie" le plan d'une "proaction" ou "proréaction". Une "proaction" est spontanée, une "proréaction" est amorcée par une situation extérieure.

31 Des 60 documents choisis, nous en avons pris 18 qui avaient pour auteur Mgr Louis-François Laflèche, qui fut évêque des Trois-Rivières. Ces dix-huit documents nous ont paru rendre l'ensemble du contenu analysé plus représentatif de l'idéologie du groupement ultramontain des années 1870 et 1880 . Des documents cités par des historiens nous avaient assez démontré que durant ces décennies, l'évêque des Trois-Rivières était perçu par les autres ultramontains et les contemporains comme le leader incontesté du groupement ultramontain, sa voix autorisée et son théoricien fidèlement suivi. Il est par ailleurs historiquement certain que le prélat assuma effectivement cette triple fonction. 
sentent une dizaine de membres certains du groupement ultramontain qui, selon toute vraisemblance, y ont détenu les statuts les plus élevés. Tous les documents choisis traitent d'interactions antagoniques qui se sont produites entre des ultramontains et des membres du groupement "libéral" canadien-français, ce groupement que les ultramontains percevaient contestataire des normes sociales qu'il voulaient maintenir institutionnalisées. En outre, étant donné leurs destinataires et leurs fonctions respectives, les 60 documents choisis, avons-nous pensé au début de notre recherche, nous révéleraient peut-être des contenus de plans, croyances et aperceptions de personnes vraisemblablement évalués vrais par les ultramontains et plus ou moins contradictoires à des contenus de plans, croyances et aperceptions qu'ils auraient évalués faux. 37 de ces documents furent des messages privés dont 10 inter-ultramontains, et 33 furent des messages publics. L'intention principale (ou fonction) des auteurs de la majorité des 60 documents fut ou expressive ou conative et non pas simplement référentielle. ${ }^{32}$

\section{La technique de l'analyse thématique}

Pour mieux atteindre les deux objectifs qui viennent d'être fixés, nous avons voulu que notre observation des 60 écrits sélectionnés soit la plus systématique et objective possible et en partie quantitative. Nous nous sommes donc inspiré pour la faire des procédés de l'analyse de contenu conçus et développés surtout par les politicologues américains Bernard Berelson, Harold Laswell et Paul Lazarsfeld et par un groupe de chercheurs de l'Université d'Iowa. Nous avons fait l'analyse thématique des 60 messages, l'une des variantes de l'analyse de contenu.

Ont d'abord été relevés tous les passages des documents, généralement d'un à trois paragraphes chacun, qui, implicitement ou explicitement, révélaient des conceptions idéologiques, culturelles et stratégiques de l'in-group ultramontain. De la même façon, nous avons dressé une liste des extraits révélant des traits perçus de soi et d'autrui.

Puis chaque extrait a été réduit en un ou plusieurs thèmes - un thème est une assertion sur un sujet concret ou "référent" - Cette première uniformisation faite, nous avons regroupé les thèmes à peu près identiques et n'avons retenu que ceux, idéologiques, culturels et stratégiques, communiqués par

32 Nous donnons aux termes "expressif", "conatif" et "référentiel" le sens que leur a donné Roman Jakobson dans Essais de linguistique générale (Paris, Editions de Minuit, 1963), 209-248. 
au moins quatre ultramontains : les thèmes possédant cette fréquence de 4 nous ont paru offrir une garantie minimale qu'ils ont fait partie du super-ego de la majorité des membres du groupement ultramontain.

Nous avons ensuite défini des référents plus généraux à partir des thèmes idéologiques, culturels et tactiques récurrents quatre fois. Avec ces référents ont été formées des catégories thèmes dans lesquelles ont été fondus les thèmes récurrents. Nous n'avons retenu que les thèmes aperceptifs exprimés par au moins deux ultramontains. Leurs référents étaient des personnes ou groupes : nous n'avons donc pas eu à définir des catégories thèmes pour les regrouper.

Une fois ces dernières définies, nous avons cherché, par une analyse minutieuse et qualitative, à savoir si les ultramontains n'en avaient pas classé et corrélié l'ensemble par des opérations mentales précises.

Nous croyons avoir trouvé avec assez de certitude qu'ils le furent selon des "formes" 33 ou types simples de classement (l'inclusion, la complémentation) et de mise en relation de propositions (le syllogisme, l'antithèse). Aussi bien l'ensemble des catégories thèmes idéologiques nous paraît-il avoir été ordonné par les ultramontains pour poser un diagnostic sur un soussystème social et corrélativement sur le système total canadienfrançais. Les catégories thèmes culturelles orientant les idéologiques furent vraisemblablement classées et corréliées de manière : 1) à définir des normes sociales spécifiant les fonctions-statuts intégratives et béatificatrices complémentaires de l'Eglise et de l'Etat canadiens-français et : 2) à légitimer ces normes surtout: a) par la croyance que Dieu fut leur définiteur et a voulu les institutionnaliser dans toutes les sociétés de tous les temps; b) par un rappel de lieux et temps passés où ces normes furent actualisées. Les catégories thèmes tactiques auraient été agencées de façon à tracer, pour les membres du groupement ultramontain, le plan d'une proréaction codificatrice et socialisatrice des normes sociales qui ont orienté leur évaluation idéologique.

C. Les résultats

1. LES CATEGORIES-THEMES CULTURELLES, IDEOLOGIQUES ET STRATEGIQUES dégagées du contenu des 1967), 4 .

${ }^{33}$ Jean Piaget, Logique et connaissance scientifique (Paris, Gallimard, 
60 messages analysés, communiquées par au moins 4 émetteurs de ces messages et agencées selon leur ordination probable par des membres du groupe ultramontain canadienfrançais des années 1870 et 1880

a. Les catégories thèmes culturelles orienteuses des catégories thèmes idéologiques évaluatives

aa. Des normes sociales spécifiant les fonctions-statuts intégratives ${ }^{34}$ et béatificatrices ${ }^{35}$ complémentaires de 1' "Eglise" et de 1" "Etat" du système social canadien-français

aaa. Les obligations ou émissions ("output") intégratives complémentaires de l' "Eglise" et de l' "Etat" canadiens-français à l'endroit des membres de la société canadienne-française

- Dans sa "sphère législative" propre 1' "Etat" comme "puissance législative" de la société canadienne-française, doit être "uni et subordonné" à l" "Eglise" comme "puissance législative" de la même société.

- L' "Eglise" doit définir l'étendue de sa "sphère législative" sans rencontrer la moindre opposition de la part de l' "Etat".

— La "sphère législative" de l' "Etat" doit être le "domaine temporel".

— La "sphère législative" de 1" "Eglise" doit être le "domaine spirituel de la conscience et de la religion".

- Dans cette "sphère" doivent être compris

le "mariage",

1 ' "enseignement",

la "conscience" du citoyen canadien-français élisant ses députés.

- Le pape romain doit être le législateur suprême et infaillible de 1 "Eglise et de 1' "Etat".

aab. Les obligations ou émissions ("output") ${ }^{36}$ béatificatrices complémentaires de l' "Eglise" et de l' "Etat" canadiens-français à l'endroit des membres de la société canadienne-française

— L" "Eglise" et l' "Etat" doivent contribuer à réaliser la même fin : le "bonheur" des Canadiens français.

34 Pour une définition de cette importante fonction d'un système social total, voir Talcott Parsons, "An Outline of the Social System", 57-60.

35 Dans Fonctionnement de l'Etat (Québec, Presses de l'Université Laval, 1965), Gérard Bergeron ne conçoit pas que la fonction la plus générale de l'Etat fut conceptualisée comme béatificatrice dans la plupart des cultures historiques et l'est encore dans la majorité des cultures contemporaines.

36 Pour plus de précisions sur la conception parsonienne des relations humaines comme étant des échanges attendus, voir "An Outline of the Social System", 60-64. 
— L' "Eglise" doit réaliser leur "bonheur éternel".

- L' "Etat" canadien-français doit réaliser leur "bonheur temporel".

aac. Les droits ou réceptions ("input") de 1' "Eglise" canadiennefrançaise provenant des membres de la société canadienne-française

- L' "Eglise" doit être une société indépendante de l' "Etat" canadien-français:

elle doit définir sa "constitution et son organisation" sans l'intervention législative de ceux-ci;

notamment la "constitution et l'organisation" de ses paroisses; elle doit posséder ses territoires;

elle ne doit pas payer d'impôts à l' "Etat";

elle doit avoir ses propres tribunaux parallèlement à ceux de 1' "Etat";

dans l'exercice de leurs "fonctions sacerdotales" ou "actes spirituels", ses prêtres doivent relever de la seule juridiction de ses tribunaux.

ab. Les croyances évaluatives légitimatives des normes sociales

aba. Réification et universalisation des normes sociales

- Dieu lui-même a défini, immuablement et telles que nous les définissons, les obligations intégratives et béatificatrices de l' "Eglise" et de 1' "Etat" canadiens-français et les droits de la même "Eglise".

- Dieu a d'ailleurs défini, non moins immuablement, les mêmes obligations et droits pour 1" "Eglise" et l' "Etat" de toute "nation".

abb. Rappel de lieux et temps passés de l'institutionnalisation des normes sociales

- Ces obligations et droits de l' "Eglise" et de 1' "Etat" canadiensfrançais, tels que nous les définissons, existèrent

dans l'Empire de Constantin et celui de Charlemagne;

dans l' "état social" et la "législation" de la Nouvelle-France avant sa conquête par l'Angleterre; après la conquête, elles y furent maintenues jusqu'en 1866.

b. Les catégories thèmes idéologiques: une cognition-évaluation d'un changement amorcé d'un sous-système social canadien-français

— L" "Eglise" du Canada français est gravement menacée avant tout par le "libéralisme catholique":

il y a, quoi qu'en dise "Rome" depuis 1877, "du libéralisme catholique" au Canada depuis 1848.

- Ce "libéralisme catholique" veut subordonner l' "Eglise” à l' "Etat" des Canadiens français; 
veut "séparer" cette "Eglise" et cet "Etat";

est anticlérical;

veut enlever à l' "Eglise" son contrôle exclusif de la législation scolaire.

— L" "Eglise" est aussi menacée par le "gallicanisme", fort apparenté au "libéralisme catholique".

- L' "Eglise" du Canada français est même menacée par les francsmaçons de la province de Québec, "alliés naturels des libéraux catholiques" québécois.

- Les protestants de cette province ne menacent en rien 1" "Eglise". aussi 37 . L' "Eglise" étant menacée, le "peuple canadien-français" l'est

c. Les catégories thèmes stratégiques: un plan d'une proréaction codificatrice et socialisatrice

37 Le professeur Fernand Dumont, dans "Idéologies au Canada français, 1850-1900: quelques réflexions d'ensemble", Recherches sociographiques, 2-3 (mai-décembre 1969): 145-156, semble affirmer que le groupe de pression ultramontain ne s'est pas d'abord formé pour contrer un changement perçu en passe de se faire dans le sous-système des fonctions complémentaires de l" "Eglise" et de l" "Etat" canadiens-français. Ce groupe se serait plutôt structuré avant tout et plus encore pour arrêter un autre changement, celui du sous-système des fonctions économiques (de type rural) de l'ethnie canadienne-française. Selon M. Dumont, une enquête qu'il a codirigée démontrerait que le "thème central" de l'idéologie du groupe de pression ultramontain fut l' "agriculture", soit une prise de conscience et une évaluation négative de la désinstitutionnalisation progressive du système économique rural canadien-français. (Ce changement soussystémique aurait été d'ailleurs amorcé non par la mystérieuse, omniprésente et irréversible action de l' "infrastructure économique", mais simplement par une réaction consciente de l'ethnie dominante canadienneanglaise résidant sur le territoire laurentien, par celle aussi de quelques membres de l'ethnie américaine et peut-être par des encouragements à s'industrialiser qu'auraient prodigués aux Canadiens français le groupement libéral et quelques agronomes canadiens-français de l'époque). Nous ne voyons pas à partir de quels résultats précis de son enquête M. Dumont a pu inférer ce "thème central". Notre succincte analyse ne nous l'a guère révélé. Faut-il confondre ici encore "idéologies" et "cultures" ? La sousculture économique institutionnalisée chez Mgr Laflèche et les ultramontains était vraisemblablement "agriculturiste". Ils ont pu, à l'occasion, parler de cette sous-culture qui les motivait. Mais y eut-il chez eux, dans les années 1870 et 1880 , une prise de conscience et une peur de l'industrialisation de l'ethnie canadienne-française qui eussent été plus fortes ou égales à une prise de conscience et angoisse simultanées d'un changement éventuel dans le sous-système des fonctions de l'Eglise et de l'Etat canadiens-français ? Il est difficile de le croire, si l'on considère les résultats de notre sommaire analyse et ceux de la vaste enquête menée par les professeurs Dumont, Montminy et Hamelin. 
- Il nous faut,

par l'obtention d'une approbation officielle, par le "SaintSiège", de nos idées (idéologie) et actions,

par l' "influence", dans les campagnes électorales, d'un épiscopat et clergé canadiens-français réconciliés,

par notre presse ultramontaine,

faire élire assez de députés de notre "école ultramontaine" pour qu'ils contrôlent les pouvoirs politiques canadien et québécois et fassent changer nos législations civiles de manière à ce qu'y soient recodifiées nos conceptions des obligations et droits de l" "Eglise" et de l' "Etat" canadiens-français;

fonder notre université ultramontaine à Montréal pour $\mathrm{y}$ former une nouvelle élite ultramontaine dans la société canadienne-française.

2. EXPLICITATION DES CATEGORIES THEMES culturelles, idéologiques et stratégiques étayée d'extraits des messages analysés logique

a) Les normes et croyances orientant l'évaluation idéo-

Si l'on en juge par la fréquence des thèmes valorisants abstraits des écrits analysés, il semble que le contenu évaluatif de l'idéologie ultramontaine tenait d'abord à des normes qui définissaient une relation de coopération entre l'Eglise et l'Etat canadiens-français et de subordination totale de l'un à l'autre, quand chacun de ces deux groupements exerçait sa fonction-statut de législateur de la société canadienne-française. Cette norme sociale, les ultramontains l'exprimaient sans ambages :

A l'époque de la conquête (...) la législation du Canada était encore bonne. (...) L'Etat reconnaissait l'Eglise comme une vraie société, indépendante de la société civile. L'Eglise avait son pouvoir législatif, judiciaire et coercitif, et l'exerçait librement. L'Etat lui était uni et subordonné, et reconnaissait que c'était pour lui un devoir de protéger la liberté et l'indépendance de l'Eglise. Et bien que les faux principes du faux gallicanisme eussent vicié cette législation si chrétienne dans son origine, néanmoins l'Eglise était toujours reconnue comme une société indépendante, ayant le pouvoir de faire des lois, de juger et d'infliger des peines. (Mgr Louis-François Laflèche, 1882) ${ }^{38}$

38 Le lecteur soucieux de connaître les références exactes des extraits cités dans cet article voudra bien consulter notre thèse. Là-même on trouvera en plus une critique des conclusions de l'Histoire économique et sociale du Québec, 1760-1850 du professeur Fernand Ouellet. 
Ce qu'ils entendaient par ce rapport d'union et de subordination qui devait exister entre l'Eglise et l'Etat "comme puissances législatives", les ultramontains le répétaient à souhait. Chacune de ces puissances, disaient-ils, devait avoir sa "sphère législative" propre. Celle de l'Eglise serait "le domaine spirituel de la conscience et de la religion", celle de l'Etat "le domaine temporel". Il appartiendrait à l'Eglise seule de définir les limites de sa sphère. Elle pourrait au besoin et quand bon lui semblerait s'ingérer dans la sphère de l'Etat pour censurer ses législations. Dans les textes analysés les ultramontains ne définissaient pas le domaine temporel de l'Etat. Et ils ne précisaient pas trop le domaine spirituel de l'Eglise: il leur suffisait de répéter que devraient y être inclus le "mariage", l' "enseignement" et les "fonctions sacerdotales" ou "actes spirituels" du prêtre, nommément la direction de la conscience du citoyen élisant ses députés.

Le concile de Trente avait déclaré que l'Eglise seule a le droit de faire des empêchements dirimants, de prescrire la forme du mariage ei de juger les causes mairimoniales. Dans notre nouvelle législation, l'Etat s'arroge le droit: $1^{\circ}$ de faire des empêchements dirimants de mariage, $2^{\circ}$ de prescrire la forme du mariage, $3^{\circ}$ de juger les causes matrimoniales. ${ }^{39}$

En plus de cette sujétion générale de la fonction législative de l'Etat à celle de l'Eglise, et de l'autonomie législative de celleci dans des secteurs de juridiction précis, les ultramontains auraient fortement souhaité qu'elle soit une société indépendante de l'Etat.

Dans les textes étudiés, Mgr Laflèche substitua à quelques reprises le terme "clergé" au mot "Eglise" quand il exprima ce désir. Ainsi le fit-il, par exemple :

Nous savons bien que les honorables Juges en agissant ainsi, prétendent ne juger que les abus dont le prêtre peut se rendre coupable en chaire ou ailleurs dans l'exercice du saint ministère, et qu'ils se croient obligés au nom de l'Etat, de protéger les citoyens contre l'oppression du Clergé, comme si l'Etat avait reçu la mission de gouverner l'Eglise. (Idem, 1882)

Ce fut d'ailleurs toujours dans ce sens de "clergé canadienfrançais" que les ultramontains parurent employer le mot "Eglise" dans les soixante documents analysés.

D'autre part, les ultramontains le répétaient, l'existence d'une société indépendante de l'Etat voulue pour l'Eglise supposait d'abord que l'Etat ne l'empêche en rien de "définir sa consti-

${ }^{39}$ Idem, 1881 et 1882. 
tution et son organisation" par son droit canonique. Elle impliquait aussi que l'Etat ne dépossède pas l'Eglise de ses territoires, l'exempte de l'impôt et lui accorde "l'immunité ecclésiastique", c'est-à-dire le privilège pour les clercs de ne relever que de la juridiction des tribunaux ecclésiastiques et d'échapper à celle des tribunaux civils. ${ }^{40}$

Selon les ultramontains, il eût été bien aussi que le pape de Rome exerçât le rôle de premier législateur absolu et infaillible de l'Eglise et de l'Etat canadiens-français:

Notre législation nouvelle et nos hommes d'Etat libéraux ne reconnaissent pas l'Eglise comme une société indépendante. Dans la pratique, ils ne reconnaissent pas au Souverain Pontife le droit de faire des lois qui obligent le gouvernement. $\mathrm{Si}$ ceux qui nous gouvernent reconnaissaient au Pape ce droit, ils se soumettraient à lui, et au lieu de maintenir et de défendre leur législation, ils en effaceraient tout ce qui est contraire au Syllabus. (Idem, 1882)

Telles furent les normes fondamentales qui, vraisemblablement, ont orienté l'idéologie des ultramontains canadiens-français des années 1870-1890.

L'analyse thématique a de plus indiqué qu'ils auraient particulièrement eu présentes à l'esprit, pour légitimer ces normes, trois propositions métaphysiques. L'une eût été que l'Eglise et l'Etat sont deux sociétés établies pour réaliser deux étapes hiérarchisées d'une même fin, le bonheur de l'homme. Les deux autres propositions auraient été que l'Eglise est une société parfaite dont la constitution et l'organisation sont d'origine divine alors que l'Etat est société imparfaite et organisée par l'homme. L'évêque des Trois-Rivières exprimait ainsi ces "axiomes" :

$1^{\circ}$ L'Eglise est la société de l'homme avec Dieu. Elle a pour mission de faire connaître à tous les hommes les vérités nécessaires au salut, de leur enseigner les règles de conduite que la loi de Dieu leur impose pour se sanctifier et parvenir au Ciel, et de leur en donner les moyens. Ainsi la fin de l'Eglise est de conduire l'homme à sa fin dernière, au bonheur éternel. Elle a reçu pour cela l'infaillibilité dans l'enseignement de la vérité, et la Suprématie ou la Puissance suprême dans le gouvernement des âmes. Car la sagesse infinie, en lui assignant une fin aussi sublime, n'a pas manqué de lui donner en même temps tous les moyens nécessaires pour l'atteindre. Elle tient sa constitution et son organisation de son divin Fondateur, qui a voulu qu'elle fût une société parfaite en

40 Idem, 1881 et 1882. 
elle-même, distincte et indépendante de la société civile. Son domaine comprend l'ordre spirituel, et est aussi étendu que la terre; toutes les nations sont soumises à l'enseignement divin qu'elle est chargée de leur donner. Sa durée est celle même du monde.

$2^{\circ}$ L'Etat au contraire est la société de l'homme avec l'homme, et il a pour fin de conduire les membres qui le composent au bonheur temporel. Cette société est aussi voulue par le Créateur qui a réglé, par les lois mêmes de leur nature, que les hommes dépendraient les uns des autres, et qu'ils ne pourraient atteindre leur fin naturelle ici-bas, c'est-à-dire, le bonheur temporel, que par la force de l'association et par la soumission à l'autorité. Mais Dieu ne lui a point donné de constitution ni d'organisation déterminée comme à l'Eglise. Il en a laissé le choix à la volonté des individus qui doivent le composer, et qui peuvent en varier les formes selon les besoins des temps et des lieux. (...)

$3^{\circ}$ Ces quelques notions sur l'Eglise et l'Etat, sont des vérités évidentes par elles-mêmes; elles s'imposent à l'intelligence avec la force des axiomes; il en jaillit immédiatement une vive lumière sur la nature et la nécessité de leurs rapports. (Idem, 1881)

A la présence de ces croyances légitimatives dans la culture ultramontaine, paraît s'être ajoutée aussi celle d'une conscience historique particulière de leurs normes sociales. Deux thèmes ressortis de l'analyse spécifient ainsi pareille histoire : d'abord les ultramontains reconnaissaient une première existence de leurs valeurs dans le système social de l'Empire de Charlemagne, voire même dans celui de l'Empire de Constantin:

Quand elle en est venue à faire des concordats avec les princes temporels, elle a fait comme une bonne mère qui cède devant les exigences de ses enfants devenus trop difficiles à gouverner. Elle a fait, dans la mesure du possible, des concessions sur certains points de discipline, afin d'éviter un plus grand mal. Mais avec les grands souverains, tels que Constantin le Grand et Charlemagne, l'Eglise n'avait pas besoin de faire de concordat, parce que ces hommes de génie voyaient les choses d'assez haut pour comprendre que le premier devoir d'un Souverain est d'aider l'Eglise dans l'accomplissement de sa sublime mission, qui est d'enseigner aux hommes la loi de Dieu, et de les conduire au Ciel en les sanctifiant par les sacrements. Aussi se faisaient-ils une gloire d'être les défenseurs armés de l'Eglise! (Idem, 1881)

En second lieu, ils voyaient que leurs normes sociales se trouvaient réalisées dans l'"état social" de la Nouvelle-France et s'y étaient maintenues, "après la conquête anglaise", jusqu'en 1866 . 
A l'époque de la conquête, la législation du Canada n'était autre que celle de l'ancienne monarchie française. L'Etat reconnaissait l'Eglise comme société fondée par Jésus-Christ; mais déjà les Parlements travaillaient à la dépouiller systématiquement de ses droits et de ses libertés. Et l'énergie seule des pasteurs et des fidèles aurait pu mettre alors un obstacle aux empiétements des hommes d'Etat sur les droits de l'Eglise. Néanmoins, la législation était encore bonne; il eût fallu la mettre à l'abri des influences parlementaires.

Le changement considérable que le Code Civil du Bas-Canada, adopté par la législature en 1866, a fait dans l'ancienne législation française en vigueur jusque-là, a altéré profondément les rapports de l'Eglise et de l'Etat, en subordonnant virtuellement la première au second, par les empiétements qu'il y consacre sur les choses les plus importantes, telles que le mariage, le droit de propriété, les immunités, etc., etc. (Idem, 1882)

Les ultramontains, semble-t-il, référaient fréquemment à une telle existence historique de leurs normes sociales pour ajouter une rationalisation de plus en leur faveur.

Notons enfin qu'une valeur sociétale a été aperçue deux fois dans les documents observés. Des ultramontains voulaient voir jouer un rôle nouveau à la société canadienne-française: celui de convertir au catholicisme l'Amérique anglo-saxonne protestante:

Des esprits élevés, Tardivel, Casgrain, Mgr Laflèche, élèvent très haut cet avenir du peuple canadien. D'après eux, ce peuple est le dernier-né au soleil de l'histoire; c'est le Benjamin des nations, c'est une création de la Providence qui provoque l'attention de tous les penseurs. Dieu proportionne la vocation des peuples au plan divin de son gouvernement temporel de l'humanité. L'appel tardif du peuple canadien à l'existence doit correspondre dans la suite des siècles aux desseins de Dieu sur le monde. Le dessein de Dieu c'est de régir l'humanité par son Eglise, et de faire entrer tous les peuples au sein de l'Eglise, pour assurer le maintien de la religion et en tirer la prospérité des peuples. D'après ces principes, la mission du peuple franco-canadien serait de jouer, au troisième millénaire de l'Eglise, le rôle du peuple juif dans les temps anciens, et le rôle du peuple franc dans les temps modernes. Ce rôle consiste à professer fidèlement et intégralement la vérité catholique, à la propager et à la défendre, à mettre la force au service du droit et la puissance de la nation au service de la Chaire apostolique. Evidemment cette conception est très élevée mais est-ce bien une réalité ?

(Mgr Justin Febvre, 1888 ?) 
B. La cognition-évaluation du système social canadien-français

a) Du libéralisme au gallicanisme et à la franc-maçonnerie menaçants

D'après les résultats de notre enquête, deux constatations auraient été dominantes dans la conscience qu'eurent du système social canadien-français les ultramontains de 1870 à 1890. L'une eût été qu'il existait du "libéralisme catholique" au Canada français depuis 1848, l'autre que ce libéralisme constituait la grande menace de l'Eglise au Canada. En 1877, on le sait, Rome dénia publiquement la première constatation par l'entremise d'un délégué apostolique envoyé au Canada. "Les principes pervers qui troublent l'Europe n'ont pas encore traversé l'Océan", déclara alors Mgr Conroy à son arrivée à Halifax. Après cette déclaration du prélat irlandais, les ultramontains n'en continuèrent pas moins d'affirmer l'existence du libéralisme au Canada français. Mgr Conroy l'avait faite, prétendaientils, parce que le pape, le "Saint Office" et la "Sacrée Congrégation de la Propagande" avaient été faussement renseignés par des libéraux canadiens-français. Aussi les ultramontains ont-ils multiplié démarches, mandements et mémoires, après 1877, pour convaincre les autorités romaines et les Canadiens français de l'existence du "libéralisme catholique" au Canada, qu'ils constataient de cette façon:

On s'est plu à dire et à répéter à Rome que le libéralisme canadien n'avait rien de commun avec le libéralisme européen et les premières paroles prononcées par Mgr le Délégué Apostolique en mettant le pied sur la terre canadienne prouvent que l'on avait réussi à le faire croire auprès du Saint-Siège, puisque son Excellence proclamait comme un fait certain que les principes pervers qui troublent l'Europe n'avaient pas encore traversé l'Océan. La vérité pourtant est que ces principes pervers étaient déjà acclimatés au Canada depuis plus de vingt-cinq ans (...) (Mgr LouisFrançois Laflèche, 1882)

Que le "libéralisme catholique" du Canada menaçait l'Eglise canadienne-française, des ultramontains l'affirmaient ainsi:

Si par malheur ces erreurs funestes venaient à prévaloir dans notre heureuse patrie, elles ne manqueraient pas d'attirer sur l'Eglise du Canada les mêmes calamités et les mêmes ruines qu'elles ont produites dans les diverses contrées de l'Europe. (Idem, 1882)

Grande menace de l'Eglise canadienne-française, le "libéralisme catholique" l'était, au dire des ultramontains, parce 
qu'il consistait en un ensemble de conceptions qui étaient justement l'envers de leurs normes sociales. "Subordination" de l'Eglise à l'Etat, "séparation" de l'une et de l'autre, destruction du prestige et de l'"influence" du clergé dans la société canadienne-française, liberté illimitée de la "conscience individuelle" et de son expression publique, laïcisation et étatisation de l' "école", tels étaient, pour les ultramontains, les modèles normatifs valorisés par les "libéraux catholiques": ${ }^{41}$

Tels sont, N.T.C.F., les avis importants que nous croyons devoir vous donner dans les circonstances actuelles: Défiezvous surtout de ce libéralisme qui veut se décorer du beau nom de catholique pour accomplir plus sûrement son œuvre criminelle. Vous le reconnaitrez facilement à la peinture qu'en a faite souvent le Souverain Pontife: $1^{\circ}$ Efforts pour asservir l'Eglise à l'Etat; $2^{\circ}$ tentatives incessantes pour briser les liens qui unissent les Enfants de l'Eglise entre eux et avec le clergé; $3^{\circ}$ alliance monstrueuse de la vérité avec l'erreur, sous prétexte de concilier toutes choses et d'éviter des conflits; $4^{\circ}$ enfin, illusion et quelquefois hypocrisie, qui, sous des dehors religieux et de belles protestations de soumission à l'Eglise, cache un orgueil sans mesure. (Les évêques de la Province ecclésiastique de Québec, 1875)

Dans leurs écrits, les ultramontains précisaient leur constatation de l'existence du "libéralisme catholique" au Canada français depuis 1848 par d'autres assertions réitérées. Ainsi, disaient-ils, le "libéralisme catholique" était "enraciné dans la classe gouvernante du pays".

Ses plus ardents promoteurs se trouvaient à la tête du parti libéral canadien et du parti national québécois.

Outre l'Université Laval qui professait les doctrines "libérales catholiques", une partie "influente" du clergé québécois, "les prêtres de l'Archevêché, du Séminaire et de l'Université Laval de Québec" se faisaient même les "inspirateurs" du "libéralisme catholique". Conseillé par ses prêtres, Mgr Taschereau, archevêque de Québec, donnait, lui aussi, souventes fois dans les erreurs de cette "funeste doctrine" :

Permettez-moi de le dire, Eminentissimes Seigneurs, là n'est point la première cause de nos difficultés; la véritable cause se trouve dans les influences diverses que subit à son insu, je pense bien, Mgr l'Archevêque, et qui l'inclinent tantôt à marcher avec ses suffragants, et tantôt à favoriser les libéraux. Voilà ce que tout le monde remarque et que beaucoup ne se gênent pas de dire. (Mgr Louis-François Laflèche, 1882)

41 Idem, 1882. 
A deux ou trois reprises, dans les écrits analysés, des ultramontains ont constaté avec regret l'existence de "gallicanisme" au Canada. Mais apparemment "gallicanisme" et "libéralisme" ont eu le même sens dans leur esprit: chaque fois, le gallicanisme constaté était défini comme le "libéralisme catholique".

Des ultramontains considéraient que l'Eglise du Canada français était aussi menacée par la "franc-maçonnerie". Selon eux, le nombre des francs-maçons croissait dans la province de Québec, et quoi qu'on en disait, les francs-maçons québécois ne différaient en rien de ceux d'Europe. En outre, ils étaient les "alliés naturels des libéraux" et partageaient leurs croyances et modèles.

b) De l'Eglise au peuple menacé

Menace pour l'Eglise, le "libéralisme catholique" était par le fait même une menace pour le "bon peuple canadien-français" ou la "sociétée", disaient les ultramontains :

Les plus grands ennemis du peuple sont donc ceux qui veulent bannir la religion de la politique; car sous prétexte d'affranchir le peuple de ce qu'ils appellent la tyrannie du prêtre, l'influence indue du prêtre, ils préparent à ce même peuple les chaînes les plus pesantes et les plus difficiles à secouer...

(Les évêques de la Province ecclésiastique de Québec, 1875)

Dans les textes vus, les ultramontains ont beaucoup moins fréquemment affirmé que le "libéralisme catholique" menaçait le peuple canadien-français qu'ils ne l'ont dit de l'Eglise. La première assertion ne fut de plus jamais explicitée davantage : on ne précisait pas, comme on le faisait pour l'Eglise, en quoi le peuple québécois était menacé. Même de ce que la diffusion des idées libérales dans le peuple aurait pu changer de ses interactions avec l'Eglise, de ses comportements religieux ou de ses croyances métaphysiques, on ne disait mot. Aussi n'estil pas impossible que cette allégation imprécise, étendant à tout le peuple canadien-français le péril encouru par l'Eglise, n'ait pas été perçue comme un fait réel par les ultramontains. Ils l'auraient plutôt considérée comme une rationalisation mythique, un épouvantail à brandir publiquement pour mieux institutionnaliser leurs normes sociales dans le peuple. Axée uniquement sur les relations de l'Eglise et de l'Etat comme elle paraît bien l'avoir été, l'action évaluative morale des ultramontains ne porta nullement du reste sur les interrelations des groupes du peuple entre eux ou avec l'Eglise. Ce désintéresse- 
ment ne dénote-t-il pas aussi une inconscience des problèmes du prolétariat? Mais il n'en demeurait pas moins nécessaire que les modèles ultramontains, tout clérico-centriques, soient partagés par le peuple pour être démocratiquement recodifiés par l'Etat. Et cela, le groupement ultramontain le savait bien, comme il l'a montré dans son plan d'action socio-politique.

\section{c) Le plan de proréaction ultramontain}

Des thèmes abstraits du contenu analysé, certains définissaient une série d'actions sociales et politiques assignées au groupement ultramontains. Cet ensemble d'actions redites, nous a-t-il paru, aurait été mis en relations dans la pensée ultramontaine pour former les grandes lignes du programme d'une réaction socio-politique. Celui-ci eût recherché à faire recodifier et réinstitutionnaliser les modèles ultramontains par la société canadienne-française.

L'action primordiale du projet eût été de faire prendre les pouvoirs politiques fédéral et provincial à des candidats qui auraient entièrement partagé les valeurs ultramontaines. Le "programme catholique" du premier noyau d'ultramontains a nettement défini cette action :

Il est impossible de le nier, la politique se relie étroitement à la religion et la séparation de l'Eglise et de l'Etat est une doctrine absurde et impie. Cela est particulièrement vrai du régime constitutionnel qui, attribuant au parlement tout pouvoir de législation, met aux mains de ceux qui le composent une arme à double tranchant qui pourrait être terrible. C'est pourquoi il est nécessaire que ceux qui exercent ce pouvoir législatif, soient en parfait accord avec les enseignements de l'Eglise. C'est pourquoi il est du devoir des électeurs catholiques de choisir, pour leurs représentants, des hommes dont les principes soient parfaitement sains et sûrs.

L'adhésion pleine et entière aux doctrines catholiques romaines en religion, en politique et en économie sociale, doit être la première et la principale qualification que les électeurs catholiques devront exiger du candidat catholique. C'est le critérium le plus sûr qui devra leur servir à juger les hommes et les choses. (Anonyme, 1871)

Pour que des candidats de leur "école" puissent s'emparer des pouvoirs politiques canadien et québécois, les ultramontains auraient privilégié trois moyens précis. 1) A partir de 1877, l'un eût été d'obtenir du pape une approbation officielle de leur lutte contre les libéraux canadiens-français. Après 1877, le pape refusa toujours une telle approbation aux ultramontains. Mais, 
à leurs yeux, une seule cause rendait compte des "condamnations publiques et réitérées" qu'ils encouraient de lui: des intrigants libéraux canadiens-français et romains avaient faussement renseigné le "Saint-Office" et la "Sacrée Congrégation de la Propagande" sur le libéralisme et les affaires politico-religieuses du Canada.

Ces deux congrégations de la curie romaine avaient par la suite transmis de faux renseignements au pape et refusaient d'entendre tout témoignage des ultramontains. Momentanément dupé, le Saint-Siège finirait donc tôt ou tard, disaient-ils, par leur redonner son appui, dès que le pape, si ce n'est le "SaintOffice" et la "Propagande", se laisserait informer de la "vérité".

Si les ultramontains recherchaient tant l'assentiment $d u$ pape, c'est qu'ils le jugeaient nécessaire à leur action politique. D'un côté, il ne manquerait pas d'exercer en leur faveur une forte pression morale sur le peuple d'électeurs canadiensfrançais :

Car au Canada, à l'heure présente, malgré tout ce qu'en disent les adversaires, et à l'encontre de tout ce que l'on voit en Europe, le clergé, uni comme il l'a été jusqu'à l'avènement du Métropolitain actuel, est encore tout-puissant auprès de la nation. Les adversaires ne peuvent réussir à nous faire du mal qu'en trompant les chefs comme ils l'ont fait et en nous divisant. (Idem, 1882)

2) De plus, une sanction de Rome mettrait fin à la division de l'épiscopat et du clergé canadiens-français, au moins dans leurs attitudes à afficher à l'endroit du "libéralisme catholique". Pareille division des évêques et du clergé était en effet publiquement connue et diminuait leur prestige et leur influence auprès du peuple. Or cette influence, les ultramontains l'estimaient aussi nécessaire à leur action politique. Son utilisation en période électorale était, de leur aveu, un autre moyen de faire élire des députés ultramontains:

Car il est visible et bien constaté que, lorsque le Clergé y intervient, les élections sont moins tumultueuses; qu'il y règne par conséquent plus de paix et de bonne entente; qu'il s'y commet moins d'excès d'ivrognerie et d'intempérance; que le serment y est plus respecté et moins profané; que la corruption, en vendant et achetant les suffrages, y est plus rare; que les électeurs, étant plus instruits, choisissent des candidats plus dignes et plus capables, ce qui réduit à un plus petit nombre les candidats libéraux qui, comme il a été dit plus haut, ne sont pas dignes d'avoir les suffrages des électeurs catholiques. Mais ils seraient certains de les obtenir s'ils 
pouvaient empêcher les Prêtres de s'occuper des élections comme ils ont pu s'en occuper jusqu'ici. Car tout le monde comprend qu'il est facile de tromper le peuple, quand il n'y a personne pour lui bien expliquer les questions dont il faut s'occuper. (Mgr Ignace Bourget, 1876)

Et les ultramontains le disaient sans détour, cette "salutaire" intervention des "curés" dans les élections, dénommée "influence spirituelle indue" par les libéraux, consistait à indiquer à leurs ouailles pour qui elles devaient voter afin de ne pas pécher gravement:

Il peut se présenter un candidat dont le programme soit hostile à l'Eglise, ou bien les antécédents soient tels que sa candidature soit une menace pour ces mêmes intérêts.

De même un parti politique peut être jugé dangereux, non seulement par son programme et par ses antécédents, mais encore par les programmes et les antécédents particuliers de ses chefs, de ses principaux membres et de sa presse, si ce parti ne les désavoue point et ne se sépare pas définitivement d'eux, dans le cas où ils persistent dans leur erreur après en avoir été avertis.

(...) Alors, le prêtre et l'Evêque peuvent en toute justice et doivent en toute conscience élever la voix, signaler le danger, déclarer avec autorité que voter en tel sens est un péché, que faire tel acte expose aux censures de l'Eglise. (Les évêques de la Province ecclésiastique de Québec, 1875)

3) Enfin les ultramontains regardaient l'emploi des trois ou quatre journaux que possédaient ou rédigeaient certains d'entre eux comme un autre moyen d'action important :

Quand l'ordre de se taire sur ce qu'on appelait alors les questions brûlantes, fut donné aux journaux de la Province, la cause des libéraux catholiques était déjà très compromise dans l'opinion générale, les hommes de principes l'emportaient et la bonne presse allait triompher. C'est le pressentiment de cette victoire du parti ultramontain qui engagea alors ceux des évêques que les libéraux avaient compromis, à insister si fort à Rome, pour que l'on mît fin au combat, avant que la vérité catholique eût tout pu faire rentrer dans l'ordre. Par respect pour l'autorité, la presse de principes se tut; mais les libéraux reprirent bientôt le courage et les forces qu'ils avaient perdus dans la lutte. (Les catholiques de la Province de Québec, 1879)

Le groupement ultramontain plaçait donc au premier plan de son programme d'action sur la société le contrôle par luimême des pouvoirs politiques fédéral et provincial. Ce contrôle, il le savait, lui permettait de faire changer les législations civiles de manière à ce que soient recodifiées ses valeurs. 
Mais la tactique des ultramontains semble avoir visé plus loin que la refonte des codes civils. D'aucuns disaient souvent aussi leur intention de reprendre le contrôle de l'enseignement supérieur. Ce n'est pas qu'ils espéraient débarrasser l'Université Laval de son "libéralisme" et de sa "franc-maçonnerie". Ils entendaient plutôt fonder une et même deux universités ultramontaines à Montréal :

Dans la faculté de théologie il y a cinq chaires actuellement en pleine organisation... Trois de ces Messieurs (prêtres) sont considérés comme les coryphées du libéralisme. Ils marchent à la tête de cette petite fraction du clergé de Québec qui s'intitule libérale... Tels sont les hommes qui osent demander aux catholiques de leur confier l'éducation de leurs enfants.

Or, c'est précisément pour conserver la présence et l'influence de ces professeurs auprès de la jeunesse canadienne que le Métropolitain et son Université se récrient contre le Mémoire, et qu'ils essaient, dans un procès anti-juridique, de faire condamner l'Evêque des Trois-Rivières pour avoir trop légitimement averti ie Saint-Siège ! Que ne donnent-ilis piutôt satisfaction à la conscience catholique en assainissant le personnel universitaire! (Les catholiques de la Province de Québec, 1879)

L'Université Laval a-t-elle cette pureté de doctrine, cette puissance d'enseignement, cette richesse d'œuvres, cette maîtrise souveraine, qui fasse d'elle la capitale incontestée de la science canadienne ? A d'autres de répondre. Pour nous, une université condamnée à un rôle secondaire, cela n'a pas de sens: nous voulons, pour Montréal, au regard de Québec, une indépendance absolue; nous le croyons même nécessaire pour que, de part et d'autre, une émulation généreuse et une loyale critique acrroissent, dans chaque université, les richesses de la science. Nous irons même plus loin: nous souhaitons au Canada, la fondation d'une troisième université, confiée, non plus au clergé séculier, mais aux ordres religieux, spécialement aux Jésuites, l'ordre le plus savant et le plus apte à former des hommes. Le plus urgent besoin du Canada, c'est d'avoir des hommes de foi, des hommes de cœur, des hommes d'honneur, incapables de céder aux sollicitations de la presse, de conniver à la corruption électorale et parlementaire: hommes pieux, dévoués à la patrie et qui peuvent seuls la sauver des incohérences d'à présent. (Mgr Justin Fèvre, 1888 ?)

Ainsi, les ultramontains en avaient-ils conscience, il ne suffisait pas que leurs modèles normatifs soient recodifiés par l'Etat pour être réellement bien intégrés dans la culture canadienne-française. Il leur fallait aussi et surtout arrêter la diffu- 
sion des modèles libéraux qui déjà motivaient une partie de l'élite canadienne-française. Et la meilleure façon de le faire était de former une nouvelle élite ultramontaine dans une université ultramontaine.

3. LES THÈMES EXPRIMANT DES TRAITS PERÇUS D'AUTRUI ET DE SOI et d'une fréquence minimum de 2 dans le contenu analysé

a) Autrui

Les ennemis

"Nos libéraux catholiques" ou "nos adversaires"

- sont hypocrites dans leur attitude affichée à l'égard des "droits de l'Eglise dans l'Etat"

- sont anticléricaux

- sont voltairiens

— sont relativistes à l'égard des diverses "religions"

— sont tolérants des diverses "religions"

— sont intolérants à notre égard

— sont vindicatifs à notre égard

- sont fourbes et calomniateurs dans leur action contre nous

- sont les alliés des francs-maçons dans leur action politique

"Les prêtres libéraux de l'Archevêché, de l'Université Laval et du Séminaire de Québec"

— entendent "diminuer" et même "sacrifier" totalement les "droits de l'Eglise dans l'Etat"

- influencent fortement Mgr l'Archevêque de Québec (Taschereau)

- influencent fortement le clergé, les laïcs instruits et les étudiants

- professent les pires idées libérales

- accusent les "curés" d'ignorance

- colportent des mensonges à Rome sur les "affaires religieuses du Canada" et y calomnient NN. SS. Bourget et Laflèche

- sont amis intimes de Mgr Persico, de Mgr Zitelli et du Père Brichet, "libéraux" de la "Sacrée Congrégation de la Propagande" à Rome

- avec leurs amis "libéraux" de la "Sacrée Congrégation de la Propagande", intriguent contre NN. SS. Bourget et Laflèche à Rome français

- sont amis des politiciens "libéraux" canadiens-français

- sont influencés par leurs amis politiciens "libéraux" canadiens-

"Mgr l'Archevêque de Québec" (Taschereau) 
- manque de justesse dans son jugement

- manque de constance dans ses opinions

- est "trop timide" dans ses revendications des "droits de l'Eglise dans l'Etat"

- est fortement influencé par "les prêtres libéraux de l'Archevêché, de l'Université Laval et du Séminaire de Québec”, tout particulièrement par Mgr Benjamin Paquet, son grand vicaire

- entend détruire 1' "influence" de NN. SS. Bourget et Laflèche dans l'opinion canadienne-française

- déteste les laïcs ultramontains

- agit souvent en dehors de la ligne de conduite qui lui est tracée par le "Saint-Siège"

- a voulu punir et humilier Mgr Laflèche en essayant de démembrer son diocèse

- a publié dans les journaux canadiens-français des documents très privés qu'il avait reçus du "Saint-Siège"

"L'Université Laval"

- a perdu "ỉa confiance et les sympathies" de la majorité de l'épiscopat, du clergé et des laïcs "les plus éclairés" du Canada français

- n'a pas "les sympathies" de "Montréal" testants

— a "les sympathies" des "libéraux", des francs-maçons et des pro-

"Les francs-maçons"

— sont les "alliés naturels" des "libéraux" canadiens-français

Les dupés

"Le Saint-Office et la Sacrée Congrégation de la Propagande"

- jugent que l'épiscopat et le clergé canadiens-français sont responsables des "difficultés religieuses du Canada"

- ne veulent pas se laisser renseigner par l'épiscopat canadien-français sur les "difficultés religieuses du Canada"

- sont noyautés par une "coterie libérale"

- sont informés des "affaires religieuses du Canada" par des prêtres et laïcs "libéraux" canadiens-français intimement liés à la "coterie libérale" romaine

Les neutres

"Les protestants"

- ne sont pas anticléricaux

— n'ont nullement aidé les "libéraux catholiques" canadiens-français à changer nos législations sur les "droits de l'Eglise dans l'Etat" 
- ne s'opposent nullement à nos efforts pour rétablir les anciennes législations sur les "droits de l'Eglise dans l'Etat"

Les supporteurs

"La masse de notre peuple" ou "le petit peuple canadien-français"

- deviendra inévitablement "libéral" et révolutionnaire comme les peuples européens, s'il reste sous l'influence d'un épiscopat et d'un clergé divisés

— peut devenir "libéral" s'il reste sous l'influence de la partie "libérale" des "classes élevées" canadiennes-françaises

- est bon

- a conservé la foi simple de ses pères

— est très "religieux"

— est très dévoué à l'autorité du "Saint-Siège" supportons

- "appuie" notre action en votant pour les candidats que nous

- constitue le très grand nombre des Canadiens français

"Le clergé canadien-français" ou "les curés" les campagnes électorales

"Pie IX"

- diffère d'opinion avec le "Saint-Office" et la "Sacrée Congrégation de la Propagande" sur la cause des "difficultés religieuses du Canada"

- juge que l'épiscopat et le clergé canadiens-français ne sont pas responsables des "difficultés religieuses du Canada"

- se laisse renseigner par l'épiscopat canadien-français sur les "affaires religieuses du Canada"

- approuve NN. SS. Bourget et Laflèche

b) Soi

"Nous, Ultramontains"

- sommes des "hommes de principes"

- sommes entièrement soumis aux doctrines de l'Eglise catholique et dévoués à l'autorité du "Saint-Siège"

- détenons et défendons la "vérité"

- appartenons à l' "Ecole Ultramontaine"

- appartenons aux "bonnes familles" canadiennes-françaises

nos "principes"

- appartenons au "parti conservateur" aussi longtemps qu'il partagera

- ne voulons pas diviser le "parti conservateur" 
- voulons sauvegarder à la fois "l'Eglise et la Patrie"

- voulons que l'Etat québécois respecte les droits des protestants à l'égal de ceux de l'Eglise québécoise

"Mgr Laflèche"

- est doué d'une intelligence supérieure

- possède une instruction supérieure

- est ferme dans ses opinions

- est humble et vertueux

— est "zélé" pour son clergé et ses diocésains

- est vénéré de tous

"Mgr Bourget"

- est actif

- est doux

- est "longanime"

— est "zélé" pour son clergé et ses diocésains

- est ferme dans ses convictions

- est vénéré de tous

4. LA VARIATION DIACHRONIQUE aperçue dans les thèmes culturels, idéologiques et stratégiques présents dans le contenu analysé

Nous n'avons vu aucune variation survenir dans les propositions culturelles et idéologiques émises de 1870 à 1890 dans les 60 messages observés.

En extrayant les jugements tactiques de ces messages, nous avons noté que l'idée qu'il fallait à tout prix aux ultramontains obtenir du "Saint-Siège" une approbation officielle de leur idéologie et de leur action évaluative morale ne fut émise qu'après 1877.

Une telle idée peut difficilement avoir été conçue avant cette date. La lecture de leurs écrits nous l'a en effet indiqué, avant 1877, les "programmistes" perçurent que Pie IX les approuvait et qu'il le fit même officiellement en 1876 quand il donna à leur leader Mgr Laflèche un "bref apostolique" destiné aux Canadiens et louant la lettre pastorale des évêques de la Province ecclésiastique de Québec, promulguée le 22 septembre $1875 .^{42}$

42 Le texte intégral du "Bref de Notre Saint-Père le Pape à l'Evêque des Trois-Rivières" (18 septembre 1876) se trouve in Mgr H. Têtu et l'abbé C.-O. Gagnon, Mandements, lettres pastorales et circulaires des évêques de Québec ('Québec, 1889), I: 459-461. 
Mais en 1877 et toujours par la suite, semble-t-il, le "SaintSiège" nia officiellement la proposition fondamentale de la cognition idéologique du groupement ultramontain, à savoir que le "libéralisme catholique" "existait" au Canada français depuis 1848 et qu'il se trouvait institutionnalisé dans deux partis politiques canadiens. Le "Saint-Siège" effectua ce démenti par l'envoi, en cette année 1877, d’un délégué apostolique au Canada, Mgr Conroy. Ce prélat irlandais avait mission de mettre un terme à la division des évêques canadiens et de vérifier si le "libéralisme catholique" était vraiment "implanté" au Canada. ${ }^{43}$ Dès son arrivée à Halifax il eût déclaré: "Les principes pervers qui troublent l'Europe n'ont pas encore traversé l'Océan." 44 De plus, il contraignit très vraisemblablement ${ }^{45}$ les évêques de la Province ecclésiastique de Québec d'émettre, le 11 octobre 1877, une lettre pastorale qui rétractait en partie celle qu'ils avaient envoyée le 22 septembre 1875.

La lettre pastorale précisait que l'intention de celle du 22 septembre 1875 n'avait pas été de condamner des personnes et des partis politiques, comme l'avaient cru tous ses récepteurs: car "il n'existe aucun acte pontifical condamnant un parti politique quelconque", y disaient les évêques; leur missive spécifiait en outre qu'il appartiendrait désormais à chacun de "juger selon sa conscience" quel candidat il devrait élire pour le gouverner : par là les évêques interdisaient aux curés d'indiquer à leurs ouailles, en temps d'élection, pour qui elles devaient voter et de les menacer du "refus des sacrements" si elles ne suivaient pas leurs indications ${ }^{46}$.

43 Voir Robert Rumilly, Histoire de la Province de Québec, II: 76. On sait, comme nous le rappelle $M$. Rumilly, que les instructions données par la Sacrée Congrégation à Mgr Conroy au sujet de sa mission au Canada furent tenues secrètes jusqu'au 7 octobre 1881: alors au plus fort du différend de l'Ecole de Médecine de Montréal avec l'Université Laval, Mgr Taschereau publia le texte de ces instructions dans une circulaire au clergé de la Province ecclésiastique de Québec. Voir Mandements, lettres pastorales et circulaires des évêques de Québec publiés par Mgr Têtu et l'abbé C.-O. Gagnon..., II : 274-275.

44 C'est du moins ce que rapporte Arthur Savaète dans Voix canadiennes, Vers l'abîme, IV : 142 .

45 S'appuyant sur les contenus plutôt contradictoires des lettres pastorales du 22 septembre 1875 et du 11 octobre 1877 , Robert Rumilly infère que $\mathrm{Mgr}$ Conroy dut contraindre les évêques ultramontains, notamment Mgr Laflèche, à signer cette dernière lettre de 1877. Cette inférence de M. Rumilly est communiquée dans son Histoire de la Province de Québec, II: 98-99.

46 On trouvera le texte intégral de la "Lettre pastorale des Evêques de la Province ecclésiastique de Québec" du 11 octobre 1877, ibid., II : 51-53. 
Enfin, dans un sermon à l'emporte-pièce qu'il prononça à l'église Notre-Dame de Montréal, le $1^{\text {er }}$ novembre 1877 , Mgr Conroy s'écria: "Ne vous laissez donc point entraîner (...) par ceux qui, par excès de zèle religieux ou politique, voudraient mettre en vigueur contre des personnes ou des partis des condamnations qui n'ont jamais été prononcées ..." ${ }_{47}$

5. L'ASSOCIATION ASSEZ BIEN VERIFIEE entre 1) la structure motivationnelle évaluative morale des ultramontains canadiens-français des années 1870 et 1880 et 2 ) une perception sélective d'autrui faite par certains d'entre eux dans les mêmes années.

a) Définition des variables mises en relation et de la technique d'observation de la variable dépendante

Nous connaissions bien, au début de notre recherche, la motivation évaluative morale structurée dans les personnalités des membres du groupement ultramontain canadien-français des années 1870-1890. Nous savions aussi la forte intensité de cette motivation permanente et son objet ${ }^{4} \bar{x}$. L'un des objectifis de notre recherche fut donc d'essayer de savoir s'il y eut association entre ladite motivation ( variable $_{1}$, connue et indépendante) et le choix des perceptions de personnes fait par certains membres du groupement ultramontain dans les années 1870-1890 (variable ${ }_{2}$, inconnue et dépendante). Nous pouvions connaître indirectement les perceptions choisies par l'observation de l'échantillon que nous en présentait l'ensemble des traits perçus d'autrui communiqué dans les 60 messages observés.

b) Vérification de la relation entre les variables

Constatons que la distribution de fréquence de l'échantillon des traits perçus d'autrui observé et décrit ci-dessus, soit 52 traits, est telle que tous sont

- ou des motivations, attitudes ou comportements explicitement ou implicitement dits ou très vraisemblablement perçus centrés sur la conception qu'avaient leurs percipients des obligations et droits de l'Eglise et de l'Etat canadiens-français;

- ou des qualités très vraisemblablement perçues favorables ou défavorables à cette même conception.

47 Ibid., II: 104. M. Rumilly ne donne pas la provenance de cet extrait.

48 Nous avions lu les récits qu'ont fait des interactions évaluatives morales des ultramontains Robert Rumilly, dans son Histoire de la Province de Québec, I-V, çà et là, et $\mathrm{H}$. L. Robertson, dans The Ultramontane Group in French Canada, 1867-1886, çà et là. La reconnaissance de ces interactions nous avait permis d'inférer facilement l'état motivationnel évaluatif moral qui les causait. 
Or dans l'hypothèse de l'indépendance (ou d'une absence théorique d'association) entre 1. l'état motivationnel évaluatif moral des ultramontains ayant perçu les 52 traits et 2 . leur choix de traits perçus, la fréquence des caractères de ce choix serait ainsi distribuée:

- 26 traits seraient vus comme centrés sur la conception qu'avaient leurs percipients des droits ou obligations de l'Eglise et de l'Etat du système social canadien-français, ou vus comme favorables ou défavorables à la même conception;

- 26 traits ne seraient ni vus comme centrés sur cette conception ni vus comme lui étant favorables ou défavorables.

Quantifions l'écart entre les distributions de fréquences observées et hypothétiques et la probabilité de l'association entre la variable observée et la variable indépendante connue:

$$
\begin{aligned}
& \chi^{2}=\mathrm{\Sigma} \frac{\left(\mathrm{O}-\mathrm{A}^{*}\right)^{2}}{\mathrm{~A}}=52 \\
& \mathrm{p}>.001
\end{aligned}
$$

La probabilité d'association entre les deux variables est donc maximale.

c) La validité de cette vérification

Si l'échantillon observé d'une sélection de traits perçus d'autres personnes ou groupes représente fortement ou égale peut-être la population de traits que perçurent vraiment les ultramontains des mêmes personnes ou groupes - et nous évaluons cette forte représentativité probable sans pouvoir la vérifier - , ces caractères confirment une loi stochastique que des scientifiques ont déjà relativement bien établie sur les facteurs de la sélectivité dans la perception humaine d'autrui.

6. INFERENCES PROBABLES MAIS NON VERIFIEES sur le degré de distorsion des traits perçus d'autrui et de soi et de la partie cognitive de l'idéologie

$\mathrm{Vu}$ l'état présent de la connaissance historique objective que nous en avons, nous ne pouvons vérifier le degré de distorsion: 1) de ce que les ultramontains ont dit percevoir de soi et d'autrui dans leurs 60 messages observés et: 2) de ce qu'ils y ont dit connaître des aspects du système social canadien-français qu'ils ont évalués, notamment des normes sociales des "libéraux catholiques" canadiens-français.

* O pour caractères obtenus au terme de l'observation empirique; A pour caractères attendus dans l'hypothèse de l'indépendance d'association entre les deux variables observées. 
Néanmoins, certains de leurs propres traits furent probablement donnés par les ultramontains comme des attributs les différenciant des adversaires des normes sociales qu'ils voulaient maintenir institutionnalisées. Dans leurs recherches sur le phénomène de stéréotypage, Julian Hochberg et Henri Tajfel ont trouvé cette tendance qu'ont les personnes ou les groupes à accentuer les différences entre leurs propres traits et les traits comparables qu'ont d'autres personnes ou groupes dont ils croient se différencier par certains autres attributs (v.g., valeurs, motivations, langues, etc...) ${ }^{49}$. En inférant, sans l'avoir vérifié, que cette tendance fut probablement aussi celle des ultramontains canadiens-français du XIX ${ }^{\mathrm{e}}$ siècle, les traits autres que les valeurs et motivations par lesquels ils auraient cru se différencier des "libéraux catholiques" seraient exagérés.

Les conclusions de recherches des psychologues sociaux Secord et Backman voulant qu'une tendance à la projection existe généralement dans le processus de la perception d'autrui ${ }^{50}$ permettent d'inférer, sans pouvoir le vérifier, que les ultramontains se sont probablement vus eux-mêmes tout aussi défavorables aux personnes et groupes qu'ils percevaient défavorables à eux-mêmes.

7. NOTRE TYPOLOGIE des motivations évaluatives morales intégratives de systèmes sociaux et les types des motivations évaluatives morales $\mathrm{du}$ groupement ultramontain canadienfrançais

\section{a) Notre typologie}

Nous avons utilisé le cadre conceptuel parsonien des systèmes sociaux ${ }^{51}$ pour former une typologie des motivations évaluatives morales intégratives de systèmes sociaux. Nous concevons que ce type de motivation pourra être, selon les cas empiriques, de l'un ou de l'autre, de certains ou de tous les sous-types suivants:

- valeur-centrique, i.e., centrée sur la redéfinition ou le maintien de valeur sociétale

49 Julian E. Hochberg, "Psychophysics and Stereotype in Social Perception", in Conference in Social Psychology, University of Oklahoma, 1954, Emerging Problems in Social Psychology, 117-141. Aussi Henri Tajfel, "Quantity Judgment in Social Perception", British Journal of Psychology, 50 (1959) : 16-29.

50 Paul F. Secord et Carl W. Backman, Social Psychology (New York, McGraw-Hill, 1964), chap. 2 surtout. rôle.

51 Notamment ses concepts opératoires de valeur, norme, collectivité et 
- altéro-fonction-statut-obligation-centrique, i.e., centrée sur la redéfinition ou le maintien d'obligation ("output") de fonction-statut-d'autre à l'égard d'autre que le ou les redéfiniteurs ou mainteneurs

- altéro-fonction-statut-droit-centrique, i.e., centrée sur la redéfinition ou le maintien de droit ("input") de fonctionstatut d'autre à être acquittés par autre que le ou les redéfiniteurs ou mainteneurs

- ego-fonction-statut-obligation-centrique, i.e., centrée sur la redéfinition ou le maintien d'obligation de fonction-statut du ou des redéfiniteurs ou mainteneurs

- ego-fonction-statut-droit-centrique, i.e., centrée sur la redéfinition ou le maintien de droit de fonction-statut du ou des redéfiniteurs ou mainteneurs

- altéro-collectivité-centrique, i.e., centrée sur la redéfinition ou le maintien de situation à laquelle le ou les redéfiniteurs ou mainteneurs ne participent pas

- ego-collectivité-centrique, i.e., centrée sur la redéfinition ou le maintien de situation à laquelle participent le ou les redéfiniteurs ou mainteneurs

- altéro-rôle-statut-obligation-centrique, i.e., centrée sur la redéfinition ou le maintien d'obligation de rôle-statut d'autre à l'égard d'autre que le ou les redéfiniteurs ou mainteneurs - altéro-rôle-statut-droit-centrique, i.e., centrée sur la redéfinition ou le maintien de droit de rôle-statut à être acquittés par autre que le ou les redéfiniteurs ou mainteneurs

- ego-rôle-statut-obligation-centrique, i.e., centrée sur la redéfinition ou le maintien d'obligation de rôle-statut du ou des redéfiniteurs ou mainteneurs

- ego-rôle-statut-droit-centrique, i.e., centrée sur la redéfinition ou le maintien de droit de rôle-statut du ou des redéfiniteurs ou mainteneurs

b) Les types des motivations évaluatives morales du groupement ultramontain canadien-français

L'observation du contenu de l'idéologie du groupement ultramontain et du contenu culturel qui orienta les évaluations de cette idéologie nous permet d'inférer que l'intérêt évaluatif moral des membres clercs de ce groupe fut premièrement ego-fonctionsstatuts-droits-centrique (maintien) et que l'intérêt des membres laïcs de ce groupe fut premièrement altéro-fonctions-statutsdroits-centrique (maintien).

D'autres types d'intérêts, collectifs ou individuels ont pu exister chez les membres de l'in-group ultramontain simultanément à ces intérêts évaluatifs moraux. Nous ne les avons pas recherchés. 